\title{
Efficacy of Abiraterone Acetate For High-Risk Hormone-Naïve Metastatic Prostate Cancer: A Comparison With Combined Androgen Blockade Therapy With Bicalutamide And Androgen Deprivation Therapy Alone
}

Kent Kanao ( $\nabla$ kanaok@saitama-med.ac.jp )

Saitama Medical University International Medical Center

Takayuki Takahashi

Saitama Medical University International Medical Center

Yuta Umezawa

Saitama Medical University International Medical Center

Takashi Okabe

Saitama Medical University International Medical Center

Gou Kaneko

Saitama Medical University International Medical Center

\section{Suguru Shirotake}

Saitama Medical University International Medical Center

Koshiro Nishimoto

Saitama Medical University International Medical Center

Masafumi Oyama

Saitama Medical University International Medical Center

\section{Research Article}

Keywords: metastatic hormone-naïve prostate cancer, abiraterone acetate, combined androgen blockade therapy, androgen deprivation therapy, upfront intensive therapy

Posted Date: June 23rd, 2021

DOl: https://doi.org/10.21203/rs.3.rs-618611/v1

License: (c) (1) This work is licensed under a Creative Commons Attribution 4.0 International License.

Read Full License 


\section{Abstract}

Background: The treatment landscape for men with metastatic hormone-naïve prostate cancer (mHNPC) has dramatically changed with the approval of next-generation anti-androgen drugs. We compared the treatment efficacy of abiraterone with that of combined androgen blockade (CAB) therapy and androgen deprivation therapy (ADT) alone in men with high-risk mHNPC.

Methods: In total, 146 Japanese men with high-risk mHNPC were retrospectively analyzed. As initial hormonal therapy, 30, 83, and 33 men were treated with ADT plus abiraterone (ABI group), ADT plus bicalutamide (CAB group), and ADT alone (ADT group), respectively. Treatment efficacy was compared using time to castration resistance (TTCR) and prostate-specific antigen (PSA) response among the groups. Propensity score matching analysis was also performed to adjust for baseline differences.

Results: The median (95\% confidence interval [CI]) TTCR in the ABI, CAB, and ADT groups were not reached, 10.7 (7.6-13.8) months and 11.0 (7.9-12.4) months, respectively, and it was significantly longer in the $A B I$ group than in the other groups $(p=0.0012, p=0.0008)$. In propensity score matching analysis, the median TTCR was also significantly longer in the ABI group than in the other groups (hazard ratio [HR], 0.47; 95\% Cl, 0.22-0.98; $p=0.010 ; \mathrm{HR}, 0.32 ; 95 \% \mathrm{Cl}, 0.12-0.85 ; \mathrm{p}=0.004)$. The number of men who achieved PSA levels $\leq 0.2 \mathrm{ng} / \mathrm{mL}$ after propensity score matching were significantly higher in the $A B I$ group than in the other groups.

Conclusions: Our results provide important evidence regarding the superiority of abiraterone over $C A B$ therapy and ADT alone for initial treatment for men with newly diagnosed mHNPC.

\section{Background}

In the past few years, the treatment landscape for men with metastatic castration-sensitive (mCSPC) or hormone-naïve prostate cancer (mHNPC) has dramatically changed based on results of several large, randomized, phase 3 clinical trials [1-6]. These trials have shown longer survival in men with metastatic prostate cancer when androgen-deprivation therapy (ADT) was combined with abiraterone acetate (hereafter referred to as abiraterone), enzalutamide, apalutamide, or docetaxel at the time of initial ADT administration.

In Japan, abiraterone was approved for men with high-risk mHNPC in 2018 based on the findings of the LATITUDE study, which showed a significant increase in the overall survival (OS) and radiographic progression-free survival (rPFS) in men with newly diagnosed high-risk mCSPC. However, combined androgen blockade (CAB) therapy with bicalutamide ( $80 \mathrm{mg}$ daily) had been generally used in Japanese men with mHNPC based on the results of a large multicenter randomized controlled trial [7]. Therefore, a comparison of abiraterone and bicalutamide treatment in men with mHNPC may provide information regarding the selection of initial treatment because the control arm in the LATITUDE study used ADT alone [4]. Further, to our knowledge, no study has directly compared the treatment efficacy of abiraterone and bicalutamide in men with mHNPC. 
In this study, we compared the treatment efficacy of abiraterone with that of bicalutamide and ADT alone in Japanese men with newly diagnosed high-risk mHNPC.

\section{Patients And Methods}

\section{Patients}

In this retrospective single institutional study, we identified a total of 146 men with newly diagnosed mHNPC between 2010 and 2020 who met the high-risk criteria according to the LATITUDE study [4]. Patients initially treated with radical prostatectomy or radiotherapy were excluded from this study. Clinicopathological data on age and prostate-specific antigen (PSA) level at diagnosis, clinical stage, Gleason score at diagnosis, sites of metastasis, and number of bone metastasis were obtained from the medical records of each patient. The PSA level was measured every 4-12 weeks, while radiological examinations were performed at the physician's discretion. Ethical approval for this study was granted by the ethics committee of Saitama Medical University International Medical Center (19-237). Informed consent was not required as this was a retrospective study and data were anonymized.

\section{Treatment and efficacy assessment}

As initial hormonal therapy, 30 men were treated with ADT plus abiraterone (1000 mg daily) plus prednisolone ( $5 \mathrm{mg}$ daily) (ABI group), 83 were treated with ADT plus $80 \mathrm{mg}$ bicalutamide (CAB group), and 33 men were treated with $A D T$ alone (ADT group). Men in the ABI group were administered abiraterone within 3 months of initial ADT administration, and those in the CAB group were administered bicalutamide within 1 month before initial ADT administration. Bilateral orchiectomy, luteinizing hormone-releasing hormone agonist, or gonadotropin-releasing hormone ( $\mathrm{GnRH})$ antagonist was used as ADT. However, in the ADT group, only GnRH antagonist (degarelix) was used as ADT.

Treatment efficacy was compared using time to castration resistance (TTCR) and PSA response among the $A B I, C A B$, and $A D T$ groups. TTCR was calculated from the initiation of initial ADT until the first development of castration resistance. Castration resistance was defined according to the Prostate Cancer Clinical Trials Working Group 3 [8].

\section{Statistical analysis}

The baseline characteristics of the three groups were compared using the chi-square and Kruskal-Wallis tests for categorical and continuous variables, respectively. The Kaplan-Meier method was used to estimate TTCR, and differences among the groups were evaluated using the log-rank test and KruskalWallis test.

Furthermore, propensity score matching analysis was performed to adjust for baseline differences. Logistic regression models were used to calculate the propensity scores; the following parameters were considered: age, initial PSA levels, Gleason score, $\mathrm{T}$ stage (T4 or $\leq \mathrm{T} 3$ ), $\mathrm{N}$ stage (N1 of N0), and visceral metastases (yes/no). Matches were created using the most common methods, the nearest neighbor 
method, using one-to-one matching with a caliper width of 0.2. Hazard ratios (HR) and odds ratios (OR) were estimated using inverse probability of treatment weighting.

A $p$ value of $<0.05$ was considered statistically significant. All statistical analyses were performed using the statistical software R version 3.3.1. (R Core Team, 2020) and the package "Matching" and "Survival".

\section{Results}

The median (range) age and PSA level at diagnosis were 70 (47-91) years and $415(6.5-23022) \mathrm{ng} / \mathrm{mL}$. The number of men with metastasis of the extra-regional lymph node, bone, and viscera at diagnosis was 4,137 , and 34, respectively. The characteristics and differences of the three groups are summarized in Table 1. The median PSA level at diagnosis was higher in the ABI group than in the CAB and ADT group, but there were no significant differences with regard to men characteristics among the groups.

The median (range) follow-up period in the ABI, CAB, and ADT groups was 12.6 (3-90), 9.6 (1-115), and 9.6 (3-52) months, respectively. In total, 9, 69, and 27 men in the $A B I, C A B$, and ADT groups, respectively, progressed to castration-resistant prostate cancer (CRPC) during the follow-up period. The number of men with $a \geq 90 \%$ decrease in the PSA level was 30 (100\%), 75 (90\%), and $28(85 \%)$ in the ABI, CAB, and ADT groups, respectively. Further, 19 (63\%), 21 (25\%), and 1 (3\%) in the ABI, CAB, and ADT groups, respectively, achieved a PSA level of $\leq 0.2 \mathrm{ng} / \mathrm{mL}$ (Table 2 ). The median (95\% confidence interval [CI]) TTCR in the $A B I, C A B$, and ADT groups were not reached, 10.7 (7.6-13.8) months and 11.0 (7.9-12.4) months, respectively, and it was significantly longer in the $A B I$ group than in the CAB and ADT groups $(p=$ 0.0012 and $p=0.0008$, respectively; Fig. 1). However, there was no difference of the median TTCR between the CAB and ADT groups $(p=0.546)$.

In propensity score matching analysis, the median TTCR was also significantly longer in the ABI group than the CAB and ADT groups (hazard ratio [HR], 0.47; 95\% Cl, 0.22-0.98; $p=0.010$ and $\mathrm{HR}, 0.32 ; 95 \% \mathrm{Cl}$, $0.12-0.85 ; p=0.004$, respectively). However, there was no difference in the median TTCR between the CAB and ADT groups $(H R, 0.83 ; 95 \% \mathrm{Cl}, 0.53-1.3 ; \mathrm{p}=0.40)$. Figure 2 shows a comparison of the KaplanMeier curves between the three groups after propensity score matching.

A comparison of the percentage of men who achieved PSA levels $\leq 0.2$ between the three groups after propensity score matching is shown in Fig. 3 . The percentages of the $A B I$ group were significantly higher than those of the CAB and ADT groups $(\mathrm{OR}, 8.2 ; 95 \% \mathrm{Cl}, 2.8-24 ; \mathrm{p}<0.001$ and $\mathrm{OR}, 114.2 ; 95 \% \mathrm{Cl}, 13-$ $1040 ; p<0.001$, respectively). The percentage of men in the CAB group was also significantly higher than that of the ADT group (OR, $11.4 ; 95 \% \mathrm{Cl}, 1.4-90 ; \mathrm{p}=0.023)$.

\section{Discussion}

In this study, we showed that the median TTCR was significantly longer than in men with newly diagnosed high-risk mHNPC treated with abiraterone than in those treated with bicalutamide. To our 
knowledge, this is the first study to show the efficacy of abiraterone as an initial treatment for mHNPC compared to that of $\mathrm{CAB}$ with bicalutamide.

The effect of first-generation antiandrogens, such as bicalutamide, nilutamide, or flutamide, in addition to ADT has been widely debated because of conflicting data from individual clinical trials as well as tolerability and cost issues. In 2009, Akaza et al. in a double-blind randomized controlled study demonstrated that ADT plus $80 \mathrm{mg}$ bicalutamide had a statistically significant OS benefit in men with locally advanced or metastatic prostate cancer compared with ADT alone during long-term follow-up [7]. Based on the study by Akaza et al., ADT plus $80 \mathrm{mg}$ bicalutamide has been the standard initial treatment for metastatic prostate cancer in Japan [9]. Therefore, our results indicating superiority of abiraterone over CAB therapy provide an important evidence regarding the initial treatment in men with high-risk mHNPC. Furthermore, we showed that bicalutamide did not have any additional benefit over ADT. This result is consistent with the that reported in the study by Akaza et al., which showed that the difference in OS was greater in men with stage C/D1 disease; however, there was no difference among men with stage D2 disease [7]. Therefore, it is considered that bicalutamide does not provide any additional effect over ADT for men with high-risk mHNPC.

In this study, we used TTCR to evaluate the efficacy of abiraterone. OS is the most objective and convenient measure of meaningful clinical efficacy of investigational drugs. Moreover, rPFS has also been used as a surrogate for OS in several clinical trials. However, it is difficult to use OS for comparing treatments in cases where the control was historical and affected by historical differences in subsequent therapy. It is also difficult to use rPFS in retrospective studies such as our study as most men did not plan scheduled radiographic examination to evaluate disease progression before advancement to CRPC. Recently, several studies have indicated that TTCR can be a good surrogate endpoint of OS in men with metastatic prostate cancer $[10,11]$. Frees et al. showed that TTCR is a valid surrogate of OS and concluded that it should be prolonged as much as possible [10]. Miyake et al. also demonstrated that men with MCSPC who had a longer TTCR are likely to achieve a more favorable OS [11]. Therefore, we used TTCR as an endpoint to compare the treatment efficacy in men with mHNPC and we concluded that abiraterone may have a significant OS benefit compared with CAB therapy and ADT alone in men with newly diagnosed high-risk mHNPC.

We also compared the percentage of men who achieved PSA levels $\leq 0.2 \mathrm{ng} / \mathrm{mL}$ between the three groups after propensity score matching; the percentage of men who achieved PSA levels $\leq 0.2$ was significantly higher in the ABI group than in the CAB and ADT groups. A decline in PSA levels is a wellestablished predictor of prognosis in men with mHNPC treated with primary ADT [12-14]. Harshman et al. reported that a PSA level of $\leq 0.2 \mathrm{ng} / \mathrm{mL}$ at 7 months is a prognostic factor for longer OS with ADT for mHNPC irrespective of docetaxel administration [15]. Recently, Matsubara et al. in a post hoc analysis of the LATITUDE study showed that men treated with abiraterone plus ADT were 6.1 times more likely to achieve a PSA level of $<0.2 \mathrm{ng} / \mathrm{mL}$ than those receiving placebo plus ADT (55\% vs. 9\%) [16]. Our result is consistent with that reported in the study Matsubara et al. and suggests that newly diagnosed high-risk 
mHNPC men treated with abiraterone had a significantly better OS than men treated with CAB therapy and ADT.

This study had some limitations. This study is a retrospective single-institutional study with a relatively small cohort and short follow-up period. As mentioned above, this study evaluated TTCR and PSA response as the study endpoint as a surrogate for OS. However, we believe our results indicate superiority of abiraterone over CAB therapy, providing important evidence for initial treatment in men with mHNPC.

\section{Conclusion}

Men with high-risk mHNPC treated with abiraterone had significantly longer TTCR than those treated with $C A B$ therapy and ADT alone and were more likely to achieve an PSA level of $\leq 0.2$. Our results provide important evidence regarding the initial treatment in men with newly diagnosed $\mathrm{mHNPC}$.

\section{Abbreviations}

ADT: androgen deprivation therapy, CAB: combined androgen blockade, HR: hazard ratio, mCSPC: metastatic castration-sensitive, mHNPC: metastatic hormone-naïve prostate cancer, OR: odds ratios, OS: overall survival, PSA: prostate-specific antigen, rPFS: radiographic progression-free survival, TTCR: time to castration resistance

\section{Declarations}

- Ethics approval and consent to participate

Ethical approval for this study was granted by the ethics committee of Saitama Medical University International Medical Center (19-237). Informed consent was not required as this was a retrospective study and data were anonymized.

- Consent for publication

This manuscript contains no individual person's data

- Availability of data and materials

The datasets used and/or analysed during the current study are available from the corresponding author on reasonable request.

- Competing interests

The authors declare that they have no competing interests.

- Funding 
This work was supported by JSPS KAKENHI Grant Number JP 18 K07210.

- Authors' contributions

KK collected, analyzed and interpreted the data as well as was a major contributor in writing the manuscript. TT, YU, TO, GK, SS, KN, MO have contributed to the acquisition of data. All authors read and approved the final manuscript.

- Acknowledgements

Not applicable.

\section{References}

1. Armstrong AJ, Szmulewitz RZ, Petrylak DP, Holzbeierlein J, Villers A, Azad A, Alcaraz A, Alekseev B, Iguchi T, Shore ND et al: ARCHES: A Randomized, Phase III Study of Androgen Deprivation Therapy With Enzalutamide or Placebo in Men With Metastatic Hormone-Sensitive Prostate Cancer. J Clin Oncol 2019, 37(32):2974-2986.

2. Chi KN, Agarwal N, Bjartell A, Chung BH, Pereira de Santana Gomes AJ, Given R, Juarez Soto A, Merseburger AS, Ozguroglu M, Uemura $\mathrm{H}$ et al: Apalutamide for Metastatic, Castration-Sensitive Prostate Cancer. N Engl J Med 2019, 381(1):13-24.

3. Davis ID, Martin AJ, Stockler MR, Begbie S, Chi KN, Chowdhury S, Coskinas X, Frydenberg M, Hague WE, Horvath LG et al: Enzalutamide with Standard First-Line Therapy in Metastatic Prostate Cancer. N Engl J Med 2019, 381(2):121-131.

4. Fizazi K, Tran N, Fein L, Matsubara N, Rodriguez-Antolin A, Alekseev BY, Ozguroglu M, Ye D, Feyerabend S, Protheroe A et al: Abiraterone plus Prednisone in Metastatic, Castration-Sensitive Prostate Cancer. N Engl J Med 2017, 377(4):352-360.

5. James ND, Sydes MR, Clarke NW, Mason MD, Dearnaley DP, Spears MR, Ritchie AW, Parker CC, Russell JM, Attard G et al: Addition of docetaxel, zoledronic acid, or both to first-line long-term hormone therapy in prostate cancer (STAMPEDE): survival results from an adaptive, multiarm, multistage, platform randomised controlled trial. Lancet 2016, 387(10024):1163-1177.

6. Sweeney CJ, Chen YH, Carducci M, Liu G, Jarrard DF, Eisenberger M, Wong YN, Hahn N, Kohli M, Cooney MM et al: Chemohormonal Therapy in Metastatic Hormone-Sensitive Prostate Cancer. N Engl J Med 2015, 373(8):737-746.

7. Akaza H, Hinotsu S, Usami M, Arai Y, Kanetake H, Naito S, Hirao Y, Study Group for the Combined Androgen Blockade Therapy of Prostate C: Combined androgen blockade with bicalutamide for advanced prostate cancer: long-term follow-up of a phase 3, double-blind, randomized study for survival. Cancer 2009, 115(15):3437-3445.

8. Scher HI, Morris MJ, Stadler WM, Higano C, Basch E, Fizazi K, Antonarakis ES, Beer TM, Carducci MA, Chi KN et al: Trial Design and Objectives for Castration-Resistant Prostate Cancer: Updated 
Recommendations From the Prostate Cancer Clinical Trials Working Group 3. J Clin Oncol 2016, 34(12):1402-1418.

9. Hinotsu S, Akaza H, Usami M, Ogawa O, Kagawa S, Kitamura T, Tsukamoto T, Naito S, Namiki M, Hirao $\mathrm{Y}$ et al: Current status of endocrine therapy for prostate cancer in Japan analysis of primary androgen deprivation therapy on the basis of data collected by J-CaP. Jpn J Clin Oncol 2007, 37(10):775-781.

10. Frees S, Akamatsu S, Bidnur S, Khalaf D, Chavez-Munoz C, Struss W, Eigl BJ, Gleave M, Chi KN, So A: The impact of time to metastasis on overall survival in patients with prostate cancer. World J Urol 2018, 36(7):1039-1046.

11. Miyake H, Matsushita Y, Watanabe H, Tamura K, Motoyama D, Ito T, Sugiyama T, Otsuka A:

Prognostic Significance of Time to Castration Resistance in Patients With Metastatic Castrationsensitive Prostate Cancer. Anticancer Res 2019, 39(3):1391-1396.

12. Hussain M, Tangen CM, Higano C, Schelhammer PF, Faulkner J, Crawford ED, Wilding G, Akdas A, Small EJ, Donnelly B et al: Absolute prostate-specific antigen value after androgen deprivation is a strong independent predictor of survival in new metastatic prostate cancer: data from Southwest Oncology Group Trial 9346 (INT-0162). J Clin Oncol 2006, 24(24):3984-3990.

13. Hussain M, Goldman B, Tangen C, Higano CS, Petrylak DP, Wilding G, Akdas AM, Small EJ, Donnelly BJ, Sundram SK et al: Prostate-specific antigen progression predicts overall survival in patients with metastatic prostate cancer: data from Southwest Oncology Group Trials 9346 (Intergroup Study 0162) and 9916. J Clin Oncol 2009, 27(15):2450-2456.

14. Kijima T, Fujii Y, Yokoyama M, Ishioka J, Matsuoka Y, Numao N, Saito K, Koga F, Masuda H, Kawakami S et al: Prostate-specific antigen response to deferred combined androgen blockade therapy using bicalutamide predicts survival after subsequent oestrogen and docetaxel therapies in patients with castration-resistant prostate cancer. BJU Int 2012, 110(8):1149-1155.

15. Harshman LC, Chen YH, Liu G, Carducci MA, Jarrard D, Dreicer R, Hahn N, Garcia JA, Hussain M, Shevrin D et al: Seven-Month Prostate-Specific Antigen Is Prognostic in Metastatic HormoneSensitive Prostate Cancer Treated With Androgen Deprivation With or Without Docetaxel. J Clin Oncol 2018, 36(4):376-382.

16. Matsubara N, Chi KN, Ozguroglu M, Rodriguez-Antolin A, Feyerabend S, Fein L, Alekseev BY, Sulur G, Protheroe A, Li S et al: Correlation of Prostate-specific Antigen Kinetics with Overall Survival and Radiological Progression-free Survival in Metastatic Castration-sensitive Prostate Cancer Treated with Abiraterone Acetate plus Prednisone or Placebos Added to Androgen Deprivation Therapy: Post Hoc Analysis of Phase 3 LATITUDE Study. Eur Urol 2020, 77(4):494-500.

\section{Tables}

Due to technical limitations, table $1 \& 2$ is only available as a download in the Supplemental Files section.

\section{Figures}




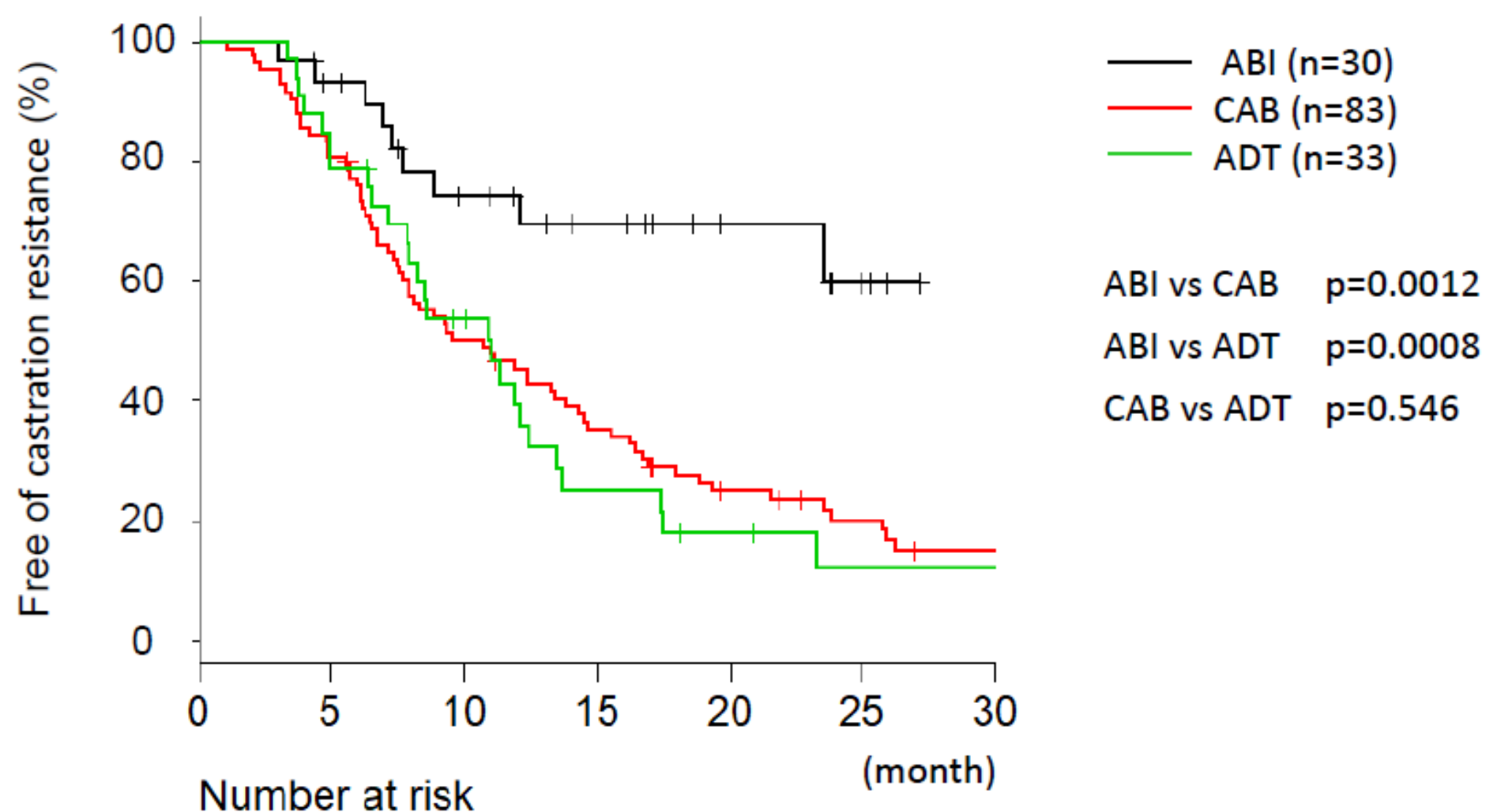

$\begin{array}{llllllll}\text { ABI } & 30 & 26 & 18 & 13 & 7 & 3 & 0 \\ \text { CAB } & 83 & 67 & 41 & 28 & 17 & 12 & 8 \\ \text { ADT } & 33 & 26 & 16 & 7 & 4 & 2 & 2\end{array}$

\section{Figure 1}

Kaplan-Meier curve comparing time to castration resistance (TTCR) among the three groups. The median TTCR in the androgen deprivation therapy (ADT) plus abiraterone (ABI group) was significantly longer than that in the combined androgen blockade (CAB) and ADT alone groups. However, there was no difference in the median TTCR between the CAB and ADT groups

$A B I$ vs $C A B$

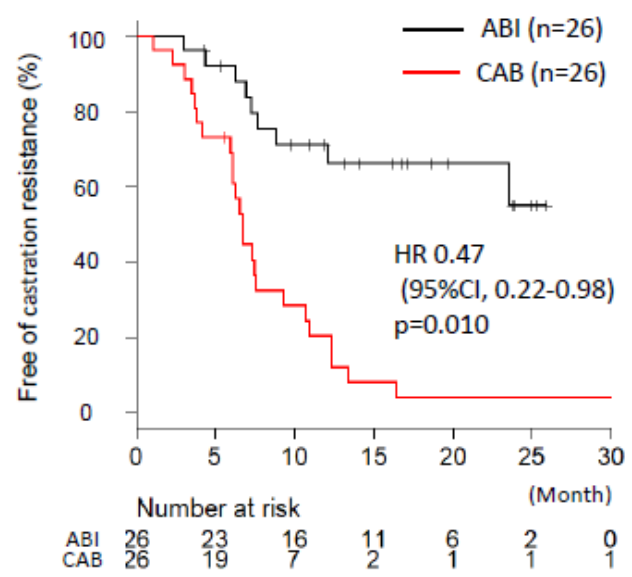

$A B I$ vs $A D T$

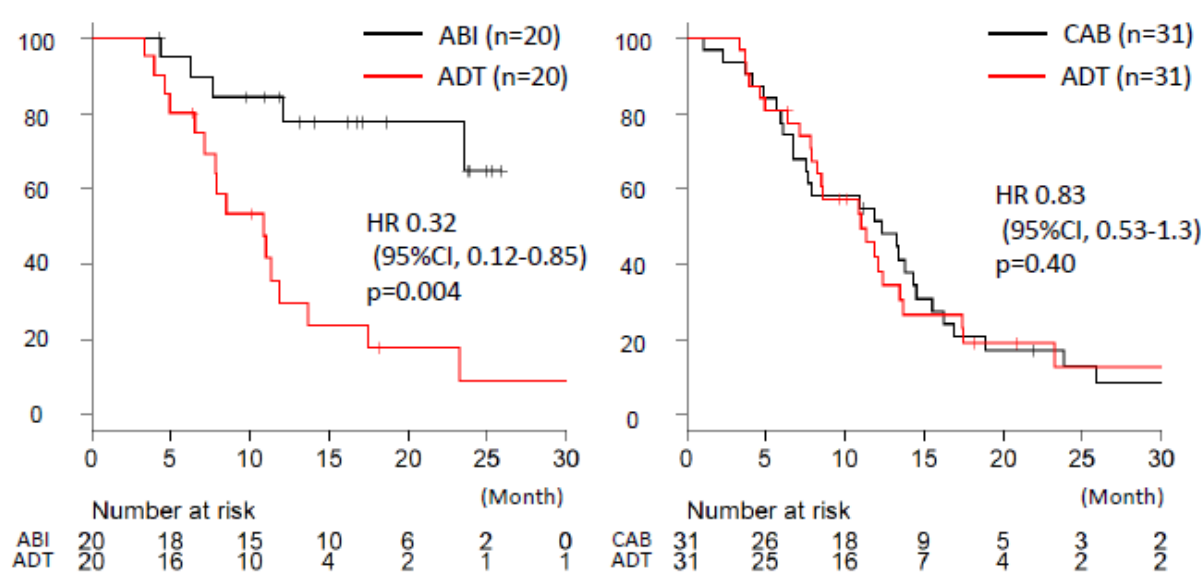

Figure 2 
Kaplan-Meier curve after propensity score matching. The median time to castration resistance (TTCR) in the androgen deprivation therapy ( $A D T$ ) plus abiraterone (ABI group) was significantly longer than that in the combined androgen blockade (CAB) and ADT alone groups. However, there was no difference of the median TTCR between the CAB and ADT groups
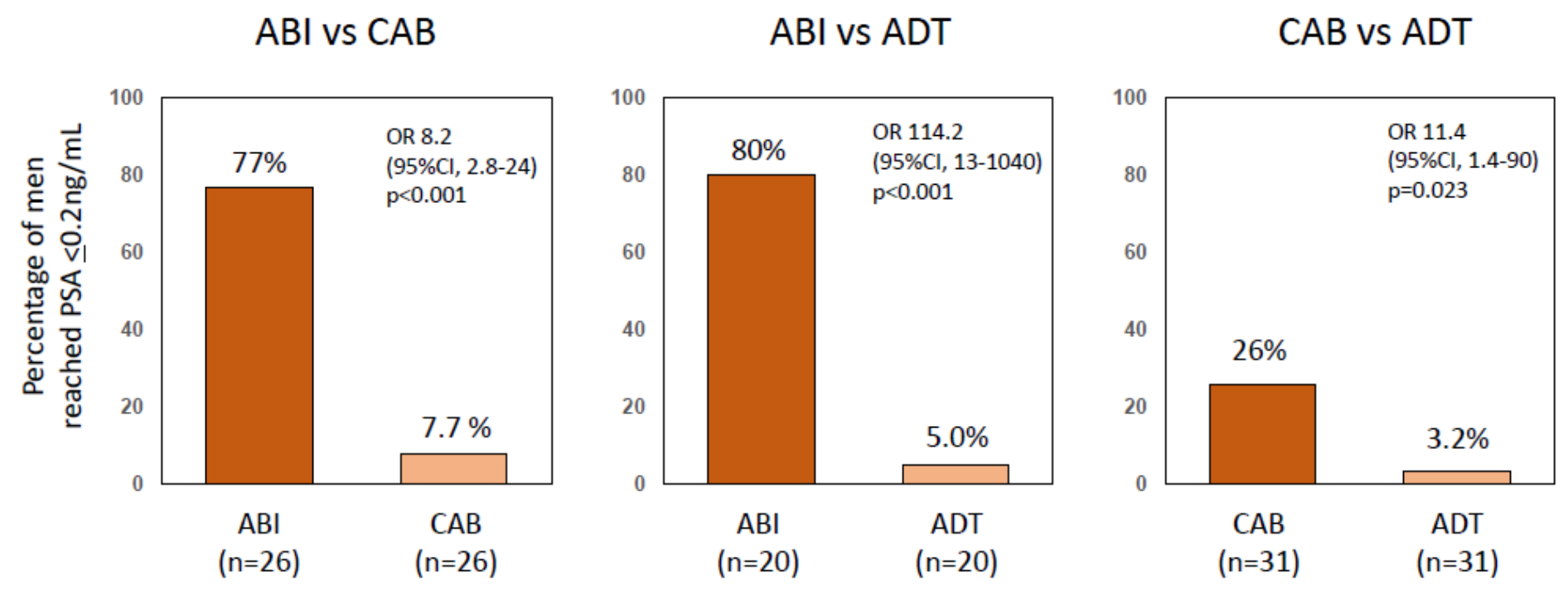

\section{Figure 3}

Percentage of men achieved a prostate-specific antigen (PSA) level of $<0.2$ after propensity score matching. The percentage of men achieved a prostate-specific antigen (PSA) level of $<0.2$ was significantly higher than in the androgen deprivation therapy (ADT) plus abiraterone ( $A B I$ group) than in the combined androgen blockade (CAB) and ADT groups

\section{Supplementary Files}

This is a list of supplementary files associated with this preprint. Click to download.

- Tablefinal.xlsx 